\title{
INTEGRAÇÃO E RELAÇÕES ÉTNICO-RACIAIS. UM EXERCÍCIO DE ANÁLISE DE REDES SOCIAIS
}

\author{
INTEGRACIÓN Y RELACIONES ÉTNICO-RACIALES. UN EJERCICIO DE \\ ANÁLISIS DE REDES SOCIALES
}

\author{
INTEGRATION AND ETHNIC-RACIAL RELATIONS. AN EXERCISE OF \\ SOCIAL NETWORKS ANALYSIS
}

\author{
Davide CARBONAI ${ }^{1}$
}

\begin{abstract}
RESUMO: O artigo examina as relações de amizade entre alunos de duas turmas de ensino médio e se propõe a destacar eventuais elementos críticos na integração entre grupos étnicos. A pesquisa utiliza algumas técnicas de análise de redes sociais: além das estatísticas de centralidade e betweeness, o texto apresenta um índice de homofilia-eterofilia, para indicar a propensão de cada aluno (Ego) a se relacionar com Alters do mesmo ou de diferente grupo étnico. Ao final, a análise aponta para algumas criticidades na integração entre grupos. Além de apresentar a pesquisa e os resultados deste exercício, o artigo introduz o leitor nos principais conceitos utilizados na análise de redes sociais, também como proposta metodológica na área de educação.
\end{abstract}

PALAVRAS-CHAVE: Análise de redes sociais. Integração social. Ensino médio. Sociologia dos grupos.

RESUMEN: El artículo examina las relaciones de amistad entre los estudiantes de dos aulas de curso secundario y tiene como objetivo tornar evidentes algunos elementos críticos en la integración de grupos étnicos. La investigación aborda algunas técnicas de análisis de redes sociales: además de las estadísticas de centralidade y betweeness, el texto presenta un índice de homofilia-heterofilia para indicar la propensión de cada estudiante (Ego) para relacionarse con Alters de el mismo o de grupo étnico distinto. Por último, el análisis apunta a una cierta criticidad en la integración entre los grupos. Además de presentar los resultados de este ejercicio, el artículo introduce al lector a los principales conceptos utilizados en el análisis de redes sociales, así como una propuesta de investigación en el campo de la educación.

PALAVRAS CLAVE: Análisis de redes sociales. Integración social. Curso secundário. Sociología de los grupos.

ABSTRACT: The paper examines friendship relations among students of two classes of middle school, and also puts in evidence some critical elements in the inter-ethnic integration process. The exercise uses social network analysis techniques: in addition to the degree and betweeness statistics, this paper presents an I-E index, to show the propensity of each student (Ego) to relate to Alters of the same or different ethnic group. Finally, the analysis points out

1 Doutor em Sociologia econômica (Università di Teramo). Atualmente é professor adjunto do Departamento de Ciências Administrativas da Universidade Federal do Rio Grande do Sul (UFRGS). Email: davide.carbonai@gmail.com. 
some critical elements of integration between groups. Over and above, in addition to main results, the paper introduces some key concepts, adopted in social network analysis.

KEYWORDS: Social network analysis. Social integration. High school. Sociology of groups.

\section{Introdução}

A análise de redes sociais adquiriu maior popularidade e consenso acadêmico ao longo do tempo: inovaram-se as técnicas, multiplicaram-se as pesquisas (em qualquer área das ciências humanas e sociais), as revistas especialistas tornaram-se mais populares (por exemplo, Social Network, Connectios, etc.), nasceram centros internacionais de pesquisa, como o INSNA (International Network for Social Network Analysis) ou brasileiros, como o GIARS (Grupo de Pesquisa Interdisciplinar em Análise em Redes Sociais da Universidade Federal de Minas Gerais).

Os primeiros usos acadêmicos da análise de redes sociais datam do início do século XX (a tríade e as geometrias sociais de Simmel, o sociograma de Moreno, por exemplo); mas é nos anos sessenta, em Manchester, que começou-se a prestar maior atenção aos laços culturalmente prescritos e àqueles realmente detectáveis; isto permitiu um avanço nas pesquisas empíricas, favorecendo, ao mesmo tempo, o desenvolvimento das técnicas e a formalização de alguns conceitos-chave: a densidade da rede, a direção, a intensidade e a reciprocidade dos arcos (MORENO, 1934; GLUCKMAN, 1965). Nos anos setenta, em Harvard, Harrison White e seu grupo de pesquisa introduziram uma abordagem mais rigorosa sob o plano estatístico-matemático; isto permitiu o último grande salto metodológico na análise de redes sociais (FORSÉ, 2002; CHIESI, 1999, pp. 36-48; WHITE et al., 1976).

A análise de redes sociais tem algumas vantagens a respeito de outras abordagens de análise: uma é de poder superar uma típica dicotomia presente nas ciências sociais, aquela entre micro e macro abordagens de análise. O princípio básico da análise de redes sociais é que a estrutura global do sistema macro é o resultado de todas as estruturas de interação micro, e vice-versa (TROBIA, 2001, p. 19-42): o micro afeta o macro, o macro afeta o micro. A análise de redes sociais concebe essa perspectiva em termos de combinação entre os «nós»- ou «vértices», ou seja, os atores incluídos na rede - e os «laços» (ou «arcos») que unem os «nós» entre si. Aqui um (quase) determinismo - aliás, questionado na literatura - pelo qual a capacidade dos 
indivíduos de influenciar a própria vida depende da estrutura de suas redes de relações sociais: o Ego-network, ou seja, o indivíduo $(E g o)$ e a relação com seus vértices adjacentes (os Alters).

Também, ao longo do tempo, o conjunto de técnicas utilizadas em análise de redes sociais tornou-se mais flexível, o que facilitou a complementariedade e a integração com outras técnicas de análise social; isto favoreceu o seu uso em várias áreas de pesquisa: por exemplo, a pesquisa organizacional, ou mais especificamente a análise de pequenos grupos de indivíduos, como no caso dos alunos de turmas escolares (CARBONAI, 2009; SMITH, 1944; MORVAL et al., 1977; FELD et al., 1982; KHMELKOV, 2002; NEWCOMB et al., 1993; WENTZEL et al., 1997).

A partir desse tipo de abordagem tornou-se possível também a análise das relações intra e inter grupos socioétnicos em contextos escolares (HALLINAN et al., 1989; DAWSON, 2008; KAWABATA, 2011; CARBONAI et al., 2014): neste caso, a análise de redes sociais destaca as diferenças estruturais entre grupos (os laços internos ao grupo e os laços externos, para outros grupos), permitindo o cálculo de algumas estatísticas globais, da rede no seu complexo, ou micro, referentes a cada síngulo (não tem significado corrente) ator incluído na rede. Isto pode ajudar na compreensão das dinâmicas ocorrentes entre alunos; de fato, como sugere George Bastin (1966, p. 12):

\begin{abstract}
A experiência mostrou as dificuldades com que lutam os professores para discernir as inter-relações que unem os alunos duma classe e descobrir as características sociais das crianças que veem todos os dias. Em certos casos, os educadores não só se sentem incapazes de consegui-lo, mas também os seus julgamentos não são concordes e invalidam, por vezes, os resultados do teste sociométrico. Este último desacordo pode justificar-se parcialmente, opondo às relações formais, determinadas pelas observações dos professores, as relações afetivas mais profundas e de caráter optativo, desvendadas pelo teste sociométrico.
\end{abstract}

A pesquisa aqui apresentada surgiu a partir de um projeto de extensão financiado em 2012 pelo Programa de Bolsas de Desenvolvimento Acadêmico (PBDA) da Universidade Federal do Pampa (Campus de São Borja). O público-alvo é representado por turmas do terceiro ano de ensino médio da Escola Estadual Padre Francisco Garcia, no município de São Borja (RS). Os questionários foram aplicados em duas turmas: turma 311 (26 alunos) e turma 312 (13 alunos). As duas redes são definidas a partir de uma pergunta-chave presente no questionário: «Quais são os teus melhores amigos? Escolha até cinco amigos, entre os colegas da tua turma: os teus preferidos». Afinal, 
trata-se de um exercício que pretende observar a integração étnico-racial, com base no sistema de laços de amizade de cada aluno entrevistado.

Ao longo do artigo são apresentados alguns dos principais conceitos utilizados em análise de redes sociais; dito isso, para aprofundar o amplo leque de técnicas ofertadas pela análise de redes sociais, sugere-se a leitura de textos clássicos na área, como o de Scott (1997) ou Wassermann e Faust (1994).

\section{A análise de redes sociais}

Em linhas gerais, a análise de redes sociais permite o cálculo de dois tipos gerais de estatísticas: o primeiro refere-se às estatísticas relativas à rede no seu conjunto (as «estatísticas macro», como por exemplo a densidade de uma rede) enquanto o segundo refere-se às estatísticas «micro», referidas a cada nó (o grau de centralidade, a betweeness, etc.). Entre as estatísticas macro, a densidade de uma rede pode ser definida pela relação entre o número de laços potenciais e os efetivamente presentes em um grafo. Para medir a densidade de um grafo simétrico (grafo não direcionado) a valores binários, é preciso utilizar a seguinte fórmula:

$$
D=\frac{l}{n(n-1) / 2}
$$

Neste caso, $l$ indica o número de laços presentes no grafo, $n$ o número de vértices. $\mathrm{O}$ denominador da fórmula indica o número máximo de laços (potencialmente presentes) no caso em que o grafo seja simétrico $(\mathrm{A} \leftrightarrow \mathrm{B})$. De fato, o cálculo da densidade - como outras estatísticas - é diferente para as redes não simétricas (tal como aquelas utilizadas neste exercício). Nos grafos direcionados (não simétricos), é provável que o nó $A$ se relacione com $B$, mas que $B$ não se relacione com $A(\mathrm{~A} \rightarrow \mathrm{B})$. A densidade é então calculada a partir de uma matriz assimétrica; para calcular a densidade neste segundo tipo de grafo, é preciso utilizar a fórmula seguinte (FREEMAN, 1979; WASSERMAN; FAUST, 1994, p. 143):

$$
D_{D I R}=\frac{l}{n(n-1)}
$$


A fórmula é bem parecida com a anterior; todavia, neste caso, o denominador não é dividido por dois (pois os laços não são simétricos).

Limitamos a análise das estatísticas micro ao caso da centralidade local (InDegree) e da betweenness. O grau de centralidade de um ator depende, em primeiro lugar, da abundância de laços de popularidade (os laços em entrada, ou seja, o número de vezes que é escolhido pelos outros nós da rede). A soma destes laços representa o grau de centralidade em entrada (também chamado de InDegree). No caso em análise, a popularidade do aluno depende da quantidade de vezes que ele é escolhido, ou seja, as escolhas dos outros vértices da rede (Alters) em relação a ele (Ego).

Este tipo de centralidade é também chamado de centralidade local, para diferenciá-la da centralidade global da betweenness: também conhecida sob o nome de estatística de «rush» ou «centralidade nos fluxos de comunicação». Neste caso, a ideia que fundamenta a estatística de betweenness é que um conjunto de hipotéticos fluxos de informações perpassaria a rede e os vértices incluídos nela: os «caminhos geodésicos», ou seja, os caminhos mais curtos que unem quaisquer duplas de vértices da rede (figura 1). A estatística de betweenness é calculada a partir da soma de todos os caminhos que cruzam o vértice $i$ (FREEMAN,1979, p.215-239).

Figura 1: Degree e betweenness (exemplo)

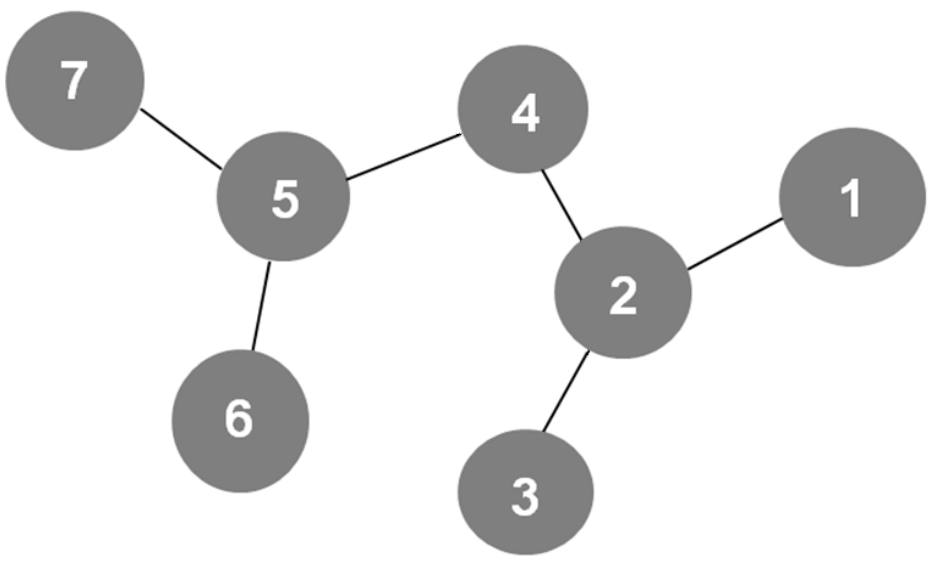

Fonte: Elaborado pelo autor

O grafo na figura 1 pode melhor exemplificar a diferença entre as duas estatísticas micro. Neste caso, os nós 5 e 2 são mais centrais em termos de Degree (o grau de centralidade local) igual a três (para ambos). No entanto, o nó 
4, apesar de não ser o mais central em termos de Degree (igual a dois), ocupa uma posição estratégica na configuração global do grafo, que permite ao nó 4 interceptar maiores «fluxos de comunicação» (ou seja, os caminhos geodésicos que juntam os outros nós da rede). Por exemplo, o nó 2 «passa» por 4 se quiser entrar em contato com o 5, 6 ou 7; igualmente se o nó 7 quiser entrar em contato com o nó 1,2 ou 3, pois passaria por 4; isto vale também para o nó 3, se quiser entrar em contato com 6 , etc.

\section{Dados e metodologia de pesquisa}

Em análise de redes sociais, é possível distinguir quatro tipos de dados, aos quais correspondem quatro tipos de matrizes sociométricas: a) as redes a valores binários e simétricos (binary non-directed graph); b) as redes a valores binários não simétricos (binary directed graph); c) redes a valores não binários simétricos (valued non-directed graph); d) redes a valores não binários assimétricos (valued directed graph). No caso desta pesquisa, são analisadas unicamente redes do segundo tipo: o aluno A pode escolher B, mas B pode não escolher A. Por isso que as matrizes de adjacência analisadas são assimétricas: os valores em entrada e saída dos nós podem ser diferentes (ver um exemplo no quadro 1).

Um conceito central na análise dos grupos é aquele de status; conforme Mucchielli (1973), o status sociométrico é representado pelo grau de consideração de um indivíduo por parte dos outros membros da rede: os Alters em relação a Ego. Ainda que limitadamente ao caso do laço de amizade, a pesquisa estuda o status do aluno (Ego). Como já apresentado, através de uma pergunta de questionário, cada aluno seleciona uma lista de colegas da própria turma, segundo um critério de preferência. Geralmente, os pesquisadores decidem considerar somente um número $x$ dos primeiros nominativos escolhidos (MORVAL et al., 1977): neste caso, os primeiros cinco. $\mathrm{Na}$ matriz estão presentes só valores binários: $O$ indica que o aluno $\mathrm{X}$ (origem do laço) não escolhe $\mathrm{Y}$ (destino do laço), 1 indica, ao contrário, que $\mathrm{X}$ escolhe $\mathrm{Y}$ na sua lista de preferências (quadro 1). 
Quadro 1: Exemplo de matriz de adjacência não simétrica (valores binários)

\begin{tabular}{c|cccccc}
\multicolumn{1}{c}{} & \multicolumn{1}{c}{ Destino do laço $(\mathrm{Y})$} \\
\cline { 2 - 6 } & & $A$ & $B$ & $C$ & $D$ & $\ldots$ \\
Origem do laço & $A$ & & 1 & 0 & 0 & $\ldots$ \\
& $B$ & 0 & & 1 & 1 & $\ldots$ \\
& $C$ & 1 & 0 & & 0 & $\ldots$ \\
& $D$ & 0 & 0 & 0 & & $\ldots$ \\
$\ldots$ & $\ldots$ & $\ldots$ & $\ldots$ & $\ldots$ &
\end{tabular}

Fonte: Elabordo pelo autor

A partir desta matriz é possível representar as relações sociais por meio de um grafo (como no caso da turma 311 na figura 2). Nesse caso, os grafos são divididos em dois grupos: os alunos que se autocolocam na classe dos «brancos» - conforme a classificação do IBGE -representados como círculos brancos, e os alunos que se autocolocam nas outras categorias (preto, pardo, amarelo e indígena) representados nos grafos com círculos cinza.

Figura 2: Grafos das relações de amizade na turma 311

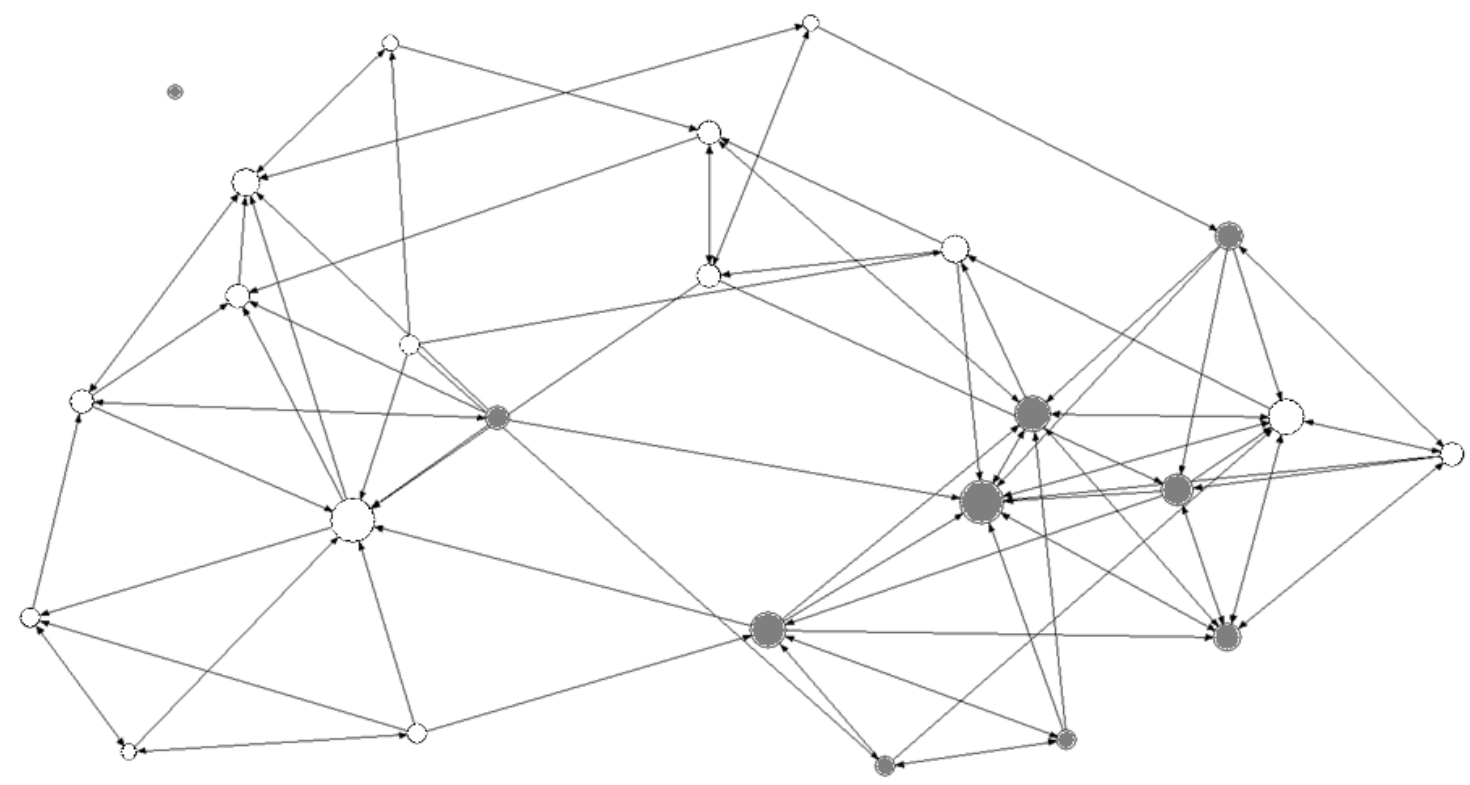

Fonte: Próprio autor

A partir desta matriz é possível calcular algumas estatísticas de rede: InDegree, Betweeness, densidade inter e intra grupal, índice de homofilia (FREEMAN, 1979, pp. 215-239) para os alunos das duas turmas, 311 (26 alunos) e 312 (13 alunos). 


\section{Principais resultados da análise de redes sociais}

As estatísticas de centralidade referidas às duas turmas podem ser observadas na tabela 2. É possível observar que no grupo 1, os brancos têm em média uma centralidade levemente menor em relação ao grupo 2 (todas as outras etnias). Todavia, neste caso, a diferença não é significativa; o teste de Kruskal-Wallis mostra um valor de p>0,05. Não muito diferente é o caso da turma 312: nesse caso a diferença não é significativa pelos valores de InDegree mas significativa no caso da betweenness $(\mathrm{p}<0,05)$. Em outros termos, em média a popularidade expressa pelo InDegree não é significativamente diferente nos dois grupos (em ambas as turmas) enquanto, no caso da betweenness, em uma turma os brancos são mais centralizados nos fluxos de comunicação que cruzam os vértices do grafo.

Tabela 2: InDegree e Betweeness nas duas turmas

\begin{tabular}{lccc} 
Turma 311 & $\mathrm{N}$ & Média & $\mathrm{p}$ \\
\hline InDegree & 25 & & \\
Grupo 1 (brancos) & 15 & 3,33 & 0,95 \\
Grupo 2 (negros) & 10 & 3,80 & \\
Betweeness & & & \\
Grupo 1 (brancos) & 15 & 42,79 & 0,59 \\
Grupo 2 (negros) & 10 & 37,10 & \\
Turma 312 & & & \\
\hline InDegree & 13 & & \\
Grupo 1 (brancos) & 5 & 3,60 & 0,45 \\
Grupo 2 (outros) & 8 & 2,75 & \\
Betweeness & & & \\
Grupo 1 (brancos) & 5 & 10,50 & 0,03 \\
Grupo 2 (outros) & 8 & 1,06 & \\
\hline
\end{tabular}

Fonte: Elaborado pelo autor

Na Figura 3, a turma 311 é dividida nos dois principais grupos: grupo 1 (os brancos) e grupo 2 (as outras etnias). Deste modo, é possível dividir a rede em quatro quadrantes, com base nas relações de origem e destino dos laços entre grupos (figura 3).

Como pode ser observado na figura 3 , mas também no grafo, o quadrante em alto à esquerda e o quadrante em baixo à direita (que representam as relações inter grupais) estão mais vazios a respeito dos outros quadrantes (que representam as relações 
intra grupais): o que representa um sinal de fechamento por parte dos grupos, que pode ser melhor pesquisado em seguida por meio de um índice que analise as relações entre grupos.

Figura 3: Densidade intra e inter grupal: turma 311

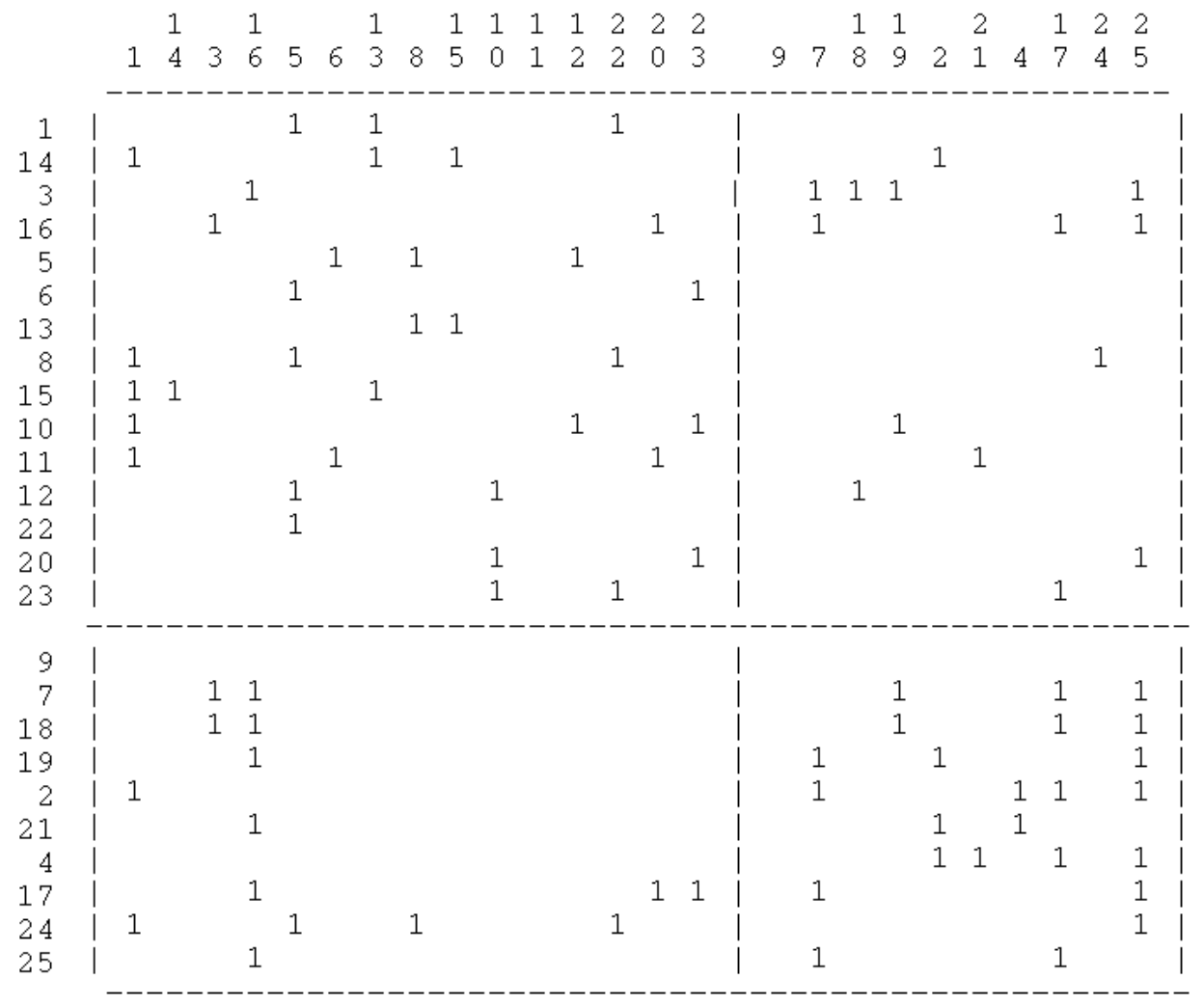

Fonte: Elaborado pelo autor

A partir desta abordagem de análise é possível calcular um índice de homofilia, para melhor entender a propensão de Ego a se relacionar com Alters do mesmo ou de outros grupos. O conceito de homofilia, em análise de redes sociais, é expresso pela diferença dos laços entre os membros do mesmo grupo (internal) de Ego e os laços externos, para vértices de outros grupos (external) (KRACKHARDT; STERN, 1988). O resultado é dividido pelo total dos laços; sendo assim, o índice varia então de 1 (máxima heterofilia) até -1 (máxima homofilia). 
Quadro 4: Índice E-I nas turmas 311 e 312

\begin{tabular}{cccccc}
\hline Turma 311 & Internal & External & Total & E-I (grupo) & E-I (turma) \\
\hline Grupo 1 & 54 & 22 & 76 & $-0,42$ & $-0,34$ \\
Grupo 2 & 36 & 22 & 58 & $-0,24$ & \\
\hline \multicolumn{7}{c}{ Turma 312 } & Internal & External & Total & E-I (grupo) & E-I (turma) \\
\hline Grupo 1 & 10 & 14 & 24 & 0,16 & 0,07 \\
Grupo 2 & 14 & 14 & 28 & 0 & \\
\hline
\end{tabular}

Fonte: Elaborado pelo autor

O índice representa um indicador válido do conceito de social closure (fechamento social) ou seu contrário, de integração social: de fato, à medida em que o índice E-I se aproxima de 1, há uma aproximação ao caso de máxima heterofilia. No caso da turma 312, o índice é substancialmente próximo de 0 , enquanto no caso da turma 311 , o índice é negativo, sinalizando um obstáculo à integração dos dois grupos ${ }^{2}$.

\section{Considerações finais}

A dificuldade de estabelecer novas formas de percepção acerca do microuniverso estudantil faz com que os educadores sejam colocados num impasse acerca do modo como agir para detectar casos de exclusão social. Nesse sentido, conforme Silva (2009):

Ao nível da estruturação das posições sociais objetivas não é possível entender os excluídos como aqueles que estão fora do sistema ou da sociedade, uma vez que a sua posição é também ela resultante dos mecanismos de funcionamento injusto do respectivo sistema social em que tais exclusões ocorrem. Ou seja, a exclusão social é segregada, em primeira e última instância, pelo próprio sistema social (SILVA, 2009, p. 55).

2 No entanto, uma vez que o índice é calculado, é importante avaliar se difere ou não de forma significativa do valor esperado. São assim lançados de forma aleatória 5000 grafos, por cada grafo, sob a hipótese que a distribuição dos laços mantenha a mesma densidade dos grafos e o tamanho dos grupos (Krackhardt; Stern, 1988). A routine em Ucinet VI restitui um valor médio diferente de forma significativa do observado no caso da turma 311, não significativo no caso da turma 312 . 
A análise de redes sociais serve então como instrumento de identificação, nos impasses encontrados em âmbito escolar, desta exclusão segregada pelo próprio sistema social (RIBEIRO, 2006); como sugere Bastin (1966, p. 15):

\begin{abstract}
Compreende-se então como é interessante para o educador e para o psicólogo, ter à sua disposição um utensílio susceptível de fornecerlhe indicações sobre a vida íntima dos grupos que educa ou examina, assim como sobre a disposição social e o papel de cada indivíduo nesses grupos.
\end{abstract}

Piaget observa que todas as formas de pesquisa social levam a um tipo de estruturalismo metodológico: a sociedade se caracteriza como uma estrutura, com suas leis de transformação e de autorregulamentação (PIAGET, 1968, p. 82). A análise de rede se torna útil para entender estruturas e explicar fenômenos do mundo real não explicados por outros tipos de análise. É reconhecido que os indivíduos não são apenas «vértices» de uma rede, mas atores complexos, com sua própria história, origem e identidade, vivem em um específico contexto social que pode influenciar, por sua natureza, o microcosmo de uma turma escolar; por sua conta, a rede oferece uma representação deste microcosmo, talvez estilizada, mas eficaz, mesmo porque simplificadora de um sistema de relações simbólicas complexo.

Afinal, como salientado por Chiesi (1999, pp. 48-75), os conceitos de «relação social», «grupo social» ou de «rede», representam um meio fundamental de explicação teórica. Logo, qualquer teoria sociológica que inclua esses conceitos precisa formalizálos: a análise de redes sociais ajuda nesta tentativa de formalização e de verificação empírica destes conceitos teóricos.

\title{
Referências
}

BASTIN, Georges. Les techniques sociometriques. Paris: Presses Universitaires de France, 1966.

CARBONAI, Davide. Valutare il networking. Note di studio sul partenariato sociale. RIV. Rivista italiana di valutazione, v. 43/44, p. 15-26, 2009.

CARBONAI, Davide; COLVERO, Ronaldo Bernardino. Papéis sociais no ensino médio: uma análise baseada na teoria das redes. Revista Brasileira de Educação, 19(58), p. 671-689, 2014.

CHIESI, Antonio M. L’analisi dei reticoli. Bologna: il Mulino, 1999. 
DAWSON, Shane. A study of the relationship between student social networks and sense of community. Educational Technology \& Society, 11(3), pp. 224-238, 2008.

FELD, Scott; ELMORE, Richard. Patterns of Sociometric Choices: Transitivity Reconsidered. Social Psychological Quarterly, 45(2), p. 77-85, 1982.

FORSE, Michel. Les réseaux sociaux chez Simmel: les fondements d'un modèle individualiste et structural, In: DEROCHE-GURCEL, Lyliane; WATIER, Patrick, La sociologie de Georg Simmel. Paris: Presses Universitaires de France, pp. 63-107, 2002.

FREEMAN, Linton C. Centrality in Social Networks: Conceptual Clarification. Social Networks, 1(3), p. 215-239, 1979.

GLUCKMAN, Max. Politics, Law and Ritual in Tribal Society. New York: The New American Library, 1965.

HALLINAN, Maureen; WILLIAMS, Richard. Interracial Friendship Choices in Secondary Schools. American Sociological Review, 54, p. 67-78, 1989.

KAWABATA, Yoshito; CRICK, Nicki R. The significance of cross-racial/ethnic friendships: Associations with peer victimization, peer support, sociometric status, and classroom diversity. Developmental Psychology, 47(6), p. 1763-1775, 2011.

KRACKHARDT, David; STERN, Robert N. Informal networks and organizational crises: an experimental simulation. Social Psychology Quarterly 51(2), pp. 123-140, 1988.

MORENO, Jacobs L. Who shall Survive? New York: Beacon House, 1934.

MORVAL, Jean; VAN GRUNDERBEECK, Nicole. Enquête sociométrique concernant le leadership et la popularité des adolescents en milieu scolaire. Revue des sciences de l'éducation, 3(1), pp. 25-35, 1977.

MUCCHIELLI, Roger. Organigrammes et sociogrammes. Paris : ESF-EME, 1973.

NEWCOMB, Andrew F.; BUKOWSKY, William; PATTEE, Linda. Children's Peer Relations: A Meta-Analytic Review of Popular, Rejected, Neglected, Controversial, and Average Sociometric Status. Psychological Bulletin, 113, pp. 99-128, 1993.

PIAGET, Jean. Le structuralisme. Paris: Presses Universitaires de France, 1968.

RIBEIRO, Carlos Antonio Costa. Classe, Raça e mobilidade social no Brasil. Dados, Rio de Janeiro, v. 49, n. 4, p. 833-873, 2006.

SCOTT, John. Social Network Analysis: A Handbook, Newbury Park, CA: Sage Publications, 1991, tr. it., L'analisi delle reti sociali, Roma: NIS, 1997.

SILVA, Manuel Carlos, Desigualdade e exclusão social: de breve revisitação a uma síntese proteórica, Configurações, 5/6, 2009, posto online no dia 15 fevereiro 2012, consultado o 22 Outubro 2013.

SMITH, Mapheus. Some factors in friendship selections of high school students. Sociometry, Vol. 7, No. 3, p. 303-310, 1944.

TROBIA, Alberto. La sociologia come scienza rigorosa. Modelli simulativi, 
intelligenza collettiva, forme del mutamento, Milano: Franco Angeli, 2001.

WASSERMAN, Stanley; FAUST, Katherine. Social network analysis: methods and applications, Cambridge: University Press Cambridge, 1994.

WENTZEL, Kathryn; CALDWELL, Kathryn. Friendships, Peer Acceptance, Group Membership: Relations to Academic Achievement in Middle School. Child Development. 68, p. 1198-1209, 1997.

\section{Como referenciar este artigo}

CARBONAI, Davide. Integração e relações étnico-raciais: um exercício de análise de redes sociais. Revista Ibero-Americana de Estudos em Educação, Araraquara/SP, v. 12, n.1, p. 205-227, 2017. Disponível em: <http://dx.doi.org/10.21723/riaee.v12.n1.8228>. E-ISSN: 1982-5587.

Data de submissão: nov/2015

Aprovação final: jan/17 